\title{
Ação da Domperidona sobre a Prenhez da Rata Albina
}

\author{
Effects of Domperidone on Pregnant Albino Rats Pregnancy
}

Arykerne Chamon do Carmo ${ }^{1}$, Danilo Nagib Salomão Paulo ${ }^{1}$, Ricardo Martins Oliveira-Filho², Manuel de Jesus Simões ${ }^{3}$, Luiz Kulay Júnior ${ }^{3}$

\section{RESUMO}

\begin{abstract}
Objetivos: estudar os efeitos do uso crônico do fármaco anti-emético domperidona durante toda a prenhez da rata albina.

Métodos: foram utilizadas 50 ratas albinas prenhes distribuidas ao acaso em cinco grupos: GI - sem nenhum tratamento (controle I); GII - recebeu água (controle II), GIII, GIV e GV foram tratadas, respectivamente, com 2, 6 e $12 \mathrm{mg} / \mathrm{kg}$ de peso corporal por dia de domperidona, com doses fracionadas de 4 em 4 horas, por gavagem, sempre em $1 \mathrm{ml}$ de água destilada, desde o dia zero até o $20^{\circ}$ dia de prenhez. O ganho de peso das matrizes foi avaliado no dia zero e no 7o, $14^{\circ}$ e $20^{\circ}$ dia de prenhez, sendo que no $20^{\circ}$ dia todos os animais foram sacrificados. A seguir foram anotados os seguintes parâmetros: número de sitios de implantação, de placentas e de fetos, pesos das placentas e dos fetos, malformações $e$ mortalidade materna e fetal.

Resultados: considerando as variáveis apreciadas, o fármaco influiu somente sobre a mortalidade fetal, sendo respectivamente de 14, 26 e 32 óbitos intra-uterinos entre 74, 60 e 57 crias, considerando os grupos III, IV e V.

Conclusões: embora os resultados da experimentação animal não possam ser diretamente levados para a clínica humana, este trabalho alerta para os cuidados na prescrição de domperidona durante o periodo gestacional.
\end{abstract}

PALAVRAS-CHAVE: Drogas na gestação. Hiperêmese. Drogas na gravidez. Óbito fetal.

\section{Introdução}

A êmese e a hiperêmese são fases evolutivas de intercorrência freqüente no início da gestação. A primeira acomete 60 a $70 \%$ das gestantes, com resolução quase sempre espontânea, constituindo a chamada "forma leve". A "forma grave", também denominada hiperêmese, acomete cerca de 2 a 3 em cada 1.000 gestações, sendo caracterizada por perda de peso, desequilíbrio eletrolítico, desidratação, cetonúria e até comprometimentos hepático, renal e neu-

\footnotetext{
${ }^{1}$ Disciplina de Obstetrícia da Escola de Medicina da Santa Casa de Misericórdia (EMESCAM), Vitória - ES.

${ }^{2}$ Departamento de Farmacologia do Instituto de Ciências Biomédicas, Universidade de São Paulo (USP), São Paulo. ${ }^{3}$ Departamento de Tocoginecologia da Universidade Federal de São Paulo/Escola Paulista de Medicina (UNIFESP/EPM). Correspondência:

Luiz Kulay Júnior

Rua Napoleão de Barros, $715-8^{\circ}$ andar

Hospital São Paulo - Disciplina de Obstetrícia

04024-002 - São Paulo - SP
}

rológico. Este tipo, embora raro no momento atual, é considerado de alto risco e necessita de internação e cuidados na área da terapia intensiva $^{1,2}$.

O esquema terapêutico para a forma leve é bastante diversificado e depende da idade gestacional, da intensidade do processo e do estado clínico da paciente. Além de eventual terapia de apoio e da dieta alimentar, é necessária prescrição medicamentosa específica para evitar agravamento dos sintomas. O arsenal terapêutico é bastante variado, estendendo-se desde vitaminas (p.ex. piridoxina) até neurolépticos (p.ex. droperidol), sempre com a premissa de não provocar teratogênese iatrogênica ${ }^{3}$.

A domperidona, potente antagonista dos receptores $\mathrm{D}_{2}$ da dopamina e com pouca repercussão sobre os seus receptores centrais, tem sido utilizada com sucesso para a normalização do peristaltismo do tubo digestivo, prevenindo inclusive vômitos de várias etiologias ${ }^{4}$.

No que concerne ao campo da reprodução, 
Marsboom ${ }^{5}$, administrando doses superiores a $40 \mathrm{mg} / \mathrm{kg}$ de peso de domperidona acrescida à alimentação balanceada, a partir do $16^{\circ}$ dia da prenhez da rata albina, observou aumento da mortalidade entre as crias.

O objetivo deste trabalho, portanto, é o de avaliar o efeito de doses diferentes da domperidona empregada durante toda a gestação sobre o binômio materno-fetal.

\section{Material e Métodos}

\section{Animais e tratamento}

Utilizamos ratas (EPM-1 Wistar), adultas, virgens, pesando de 169 a $191 \mathrm{~g}$, sob regime de alimentação (ração Purina-Labina) e de água ad libitum, que foram inicialmente acasaladas na proporção de duas fêmeas para cada macho por um periodo de duas horas. A prenhez foi diagnosticada pelo encontro de espermatozóides na luz vaginal. Cinqüenta ratas foram então divididas ao acaso em cinco grupos numericamente iguais: GI = animais sem qualquer tratamento (controle I); GII = animais que receberam água (controle II); GIII, animais tratados com $2 \mathrm{mg} / \mathrm{kg}$ por dia de domperidona; GIV, animais tratados com $6 \mathrm{mg} / \mathrm{kg}$ por dia de domperidona, e $\mathrm{GV}$, animais tratados com $12 \mathrm{mg} / \mathrm{kg}$ por dia de domperidona. O tratamento teve início logo após a positividade do teste de prenhez (dia zero) e se estendeu até o 20응 dia. Em cada caso, a dose diária foi fracionada em 3 tomadas (uma a cada 8 horas), sempre em volume total de $1 \mathrm{ml}$ de solução em cada tomada, por gavagem.

Todas as ratas foram pesadas no dia zero e no $7^{\circ}, 14^{\circ}$ e $20^{\circ}$ dia de prenhez. O ganho de peso porcentual foi calculado de acordo com a seguinte fórmula:

$\Delta \%=($ peso no dia $\underline{X}-$ peso inicial $) \times 100 /$ peso inicial

Ao termo (20 dia), todos os animais foram sacrificados por anestesia profunda com éter etílico; após laparotomia seguida de histerotomia, foram anotados os seguintes parâmetros: número de sítios de implantação, número de placentas e de fetos, pesos das placentas e dos fetos e presença de malformações. Além disto, foram registradas as mortalidades materna e fetal.

\section{Análise estatistica}

Análises paramétricas e não-paramétricas foram realizadas; o nível de rejeição para hipótese de nulidade foi fixado em $5 \%$. Comparações múltiplas foram feitas por análise de variância seguida do teste de Kruskal-Wallis.

\section{Resultados}

Não houve diferença estatisticamente significativa quanto ao ganho de peso das ratas, nos cinco grupos estudados, dois grupos controle e três grupos experimentais tratados respectivamente com 2,6 e $12 \mathrm{mg} / \mathrm{kg}$ por dia, em doses fracionadas a cada 8 horas, como pode ser visto na Figura 1 e Tabela 1.

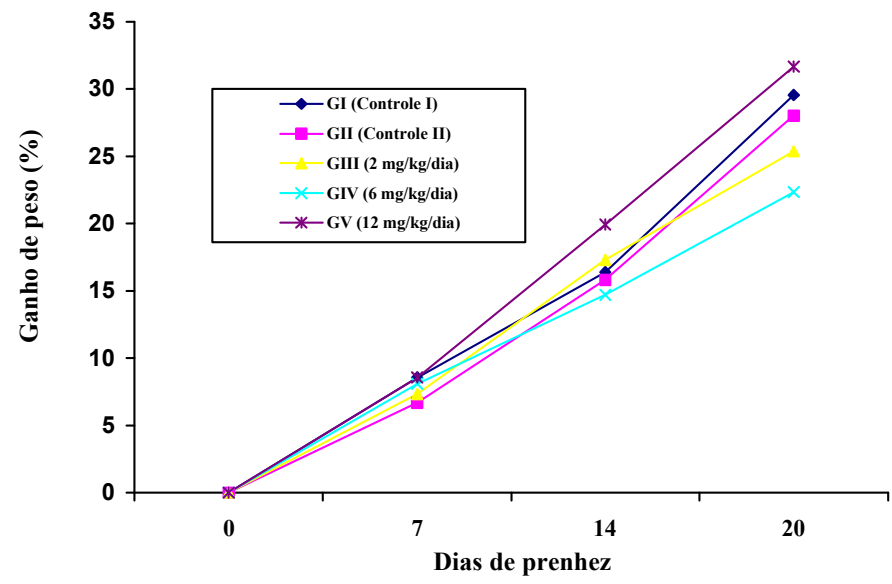

Figura 1 - Percentual de ganho de peso nos cinco grupos estudados, a partir do peso inicial, no $7^{\circ}, 14^{\circ}$ e $20^{\circ}$ dia de prenhez.

Tabela 1 - Pesos desvios-padrão (g) nos vários dias da prenhez da rata albina sob ação da domperidona.

\begin{tabular}{lcccc}
\hline & \multicolumn{4}{c}{ GRUPOS } \\
Grupos & $\mathbf{0}$ & $\mathbf{7}$ & $\mathbf{1 4}$ & $\mathbf{2 0}$ \\
\hline GI (Controle I) & $187,7 \pm 14,5$ & $203,7 \pm 16,4$ & $218,5 \pm 19,2$ & $246,0 \pm 29,9$ \\
GII (Controle II) & $177,3 \pm 22,7$ & $188,9 \pm 23,9$ & $204,7 \pm 22,7$ & $226,0 \pm 29,9$ \\
GIII $(2 \mathrm{mg} / \mathrm{kg})$ & $169,2 \pm 17,9$ & $181,5 \pm 17,3$ & $198,4 \pm 17,3$ & $211,3 \pm 25,3$ \\
GIV $(6 \mathrm{mg} / \mathrm{kg})$ & $186,1 \pm 24,7$ & $200,6 \pm 22,2$ & $212,2 \pm 20,4$ & $226,7 \pm 32,9$ \\
GV $(12 \mathrm{mg} / \mathrm{kg})$ & $191,5 \pm 38,2$ & $207,3 \pm 39,9$ & $228,7 \pm 45,4$ & $247,7 \pm 36,8$ \\
\hline
\end{tabular}


Considerando os vários parâmetros apreciados (número de implantações e de fetos, peso individual dos fetos e das ninhadas, número de placentas e peso individual e do conjunto das placentas, malformações e mortalidades materna e fetal), a única variável que demonstrou alteração significativa foi o número de natimortos, diretamente proporcional às doses administradas (Tabela 2), ou seja, 14, 26 e 32 entre 74,60 e 57 crias nos grupos III, IV e V, respectivamente.

Tabela 2 - Efeitos da domperidona sobre os vários parâmetros estudados na rata prenhe.

\begin{tabular}{lccccc}
\hline & \multicolumn{3}{c}{ GRUPOS } \\
\cline { 2 - 6 } Parâmetros & GI (controle I) & GII (controle II) & GIII (2 $\mathbf{~ g g / k g )}$ & GIV (6 $\mathbf{~ g / k g )}$ & GV (12 $\mathbf{~ m g / k g})$ \\
\hline Número de implantações & $8,7 \pm 2,2$ & $8,1 \pm 1,1$ & $7,3 \pm 2,7$ & $6,0 \pm 3,5$ & $5,6 \pm 2,8$ \\
Número de fetos & $8,7 \pm 2,2$ & $8,1 \pm 1,1$ & $7,3 \pm 2,7$ & $6,0 \pm 3,5$ & $5,6 \pm 2,8$ \\
Peso individual dos fetos & $2,3 \pm 0,6$ & $2,4 \pm 1,0$ & $2,1 \pm 0,4$ & $2,2 \pm 0,7$ & $1,8 \pm 0,1$ \\
Peso da ninhada & $20,6 \pm 6,1$ & $19,7 \pm 9,5$ & $15,6 \pm 7,2$ & $13,5 \pm 9,3$ & $10,6 \pm 4,9$ \\
Número de placentas & $8,7 \pm 2,2$ & $8,1 \pm 1,1$ & $7,3 \pm 2,7$ & $6,0 \pm 3,5$ & $5,6 \pm 2,8$ \\
Peso individual das placentas & $0,4 \pm 0,1$ & $0,5 \pm 0,1$ & $0,4 \pm 0,1$ & $0,4 \pm 0,1$ & $0,47 \pm 0,10$ \\
Peso do conjunto das placentas & $4,2 \pm 1,0$ & $4,1 \pm 1,0$ & $3,2 \pm 1,0$ & $2,7 \pm 1,5$ & $2,6 \pm 0,4$ \\
Malformações & 0 & 0 & 0 & 0 & 0 \\
Natimortos & 0 & 1 & $14^{*}$ & $26^{*}$ & $32^{*}$ \\
Mortalidade materna & 0 & 0 & 0 & 0 & 0 \\
\hline
\end{tabular}

* Significativamente diferente dos outros grupos $(p<0,006)$

\section{Discussão}

A domperidona foi ministrada a partir do dia zero até o vigésimo dia da prenhez; os conceptos, portanto, foram expostos ao fármaco durante toda a vida intra-uterina. É interessante lembrar que na rata a implantação ocorre no 4,50 dia, enquanto o período embrionário se completa no $15^{\circ}$ dia, quando se inicia o período fetal; os dois últimos estágios são caracterizados por hiperplasia, hiperplasia/hipertrofia e hipertrofia, quando ocorre o desenvolvimento dos órgãos, devido à diferenciação celular ${ }^{6}$.

Conforme mostram nossos resultados, o medicamento foi inócuo para a quase totalidade das variáveis estudadas, como ganho de peso e mortalidade materna, número de implantações e de fetos, peso individual dos fetos e das ninhadas, número de placentas e peso individual e do conjunto das placentas e malformações, quando comparadas com os grupos controle.

O mesmo, porém, não ocorreu com relação aos óbitos intra-uterinos. A mortalidade fetal foi significativamente maior nos grupos que receberam o fármaco do que aquela anotada nos grupos controle (Tabela 2). Deve ser mencionado que o número de natimortos mostrou-se diretamente proporcional às doses ministradas.

A domperidona é um antagonista dos receptores $\mathrm{D}_{2}$ da dopamina. Esta, por sua vez, sabemos que é agente precursor do metabolismo das catecolaminas e atua como neurotransmissor em determinadas regiões tanto do encéfalo quanto perifericamente ${ }^{7}$.

No sistema nervoso central a dopamina está presente no corpo estriado (parte do sistema motor extrapiramidal relacionado com a coordenação de movimentos) e no sistema límbico. Secretada diretamente dentro da circulação porta-hipofisária, atinge o lobo anterior da hipófise. Através do sistema túbero-infundibular inibe a secreção de prolactina, diminuindo ligeiramente a produção do hormônio somatotrófico, que tem pouco significado clínico ${ }^{8}$.

$\mathrm{Na}$ periferia a dopamina, substância vasoativa, contribui para otimizar o fluxo sanguíneo nos órgãos vitais. Assim, é responsável por 1) vasodilatação em rins e mesentério; 2) vasoconstrição em outros leitos vasculares, associados aos $\alpha_{1}$-adrenoceptores e 3) aumento do poder de contratilidade do miocárdio, pela ação sobre receptores $\beta$-adrenérgicos ${ }^{9-11}$. Além disto, os agonistas da dopamina atuam sobre os quimiorreceptores da "zona do gatilho" do vômito no bulbo, promovendo náuseas e vômitos ${ }^{12}$.

Portanto, a ação da domperidona, antagonizando os receptores $\mathrm{D}_{2}$, estaria restrita ao aumento da produção da prolactina, paralelamente reduzindo as náuseas e vômitos, donde sua indicação como fármaco antiemético.

É sabido que a liberação de noradrenalina 
(NAd) sofre complexa modulação em nível de membrana pré-sináptica. Assim, por exemplo, a estimulação dos receptores $\alpha_{2}$ e $\beta_{2}$-adrenérgicos pré-sinápticos causa, respectivamente, inibição e estimulação da liberação de NAd pelo terminal noradrenérgico. Além disto, em certos territórios (miocárdio e alguns leitos vasculares) ocorre importante modulação colinérgica (muscarinica) na liberação de NAd. Outros neuromoduladores também podem interferir no processo de liberação, citando-se como agentes importantes adenosina, acetilcolina, prostaglandinas, encefalinas e a dopamina, todos atuando em receptores préjuncionais ${ }^{13}$. O efeito desses compostos é provavelmente devido à inibição da função de canais de $\mathrm{Ca}^{2+}$ localizados na membrana pré-sináptica ${ }^{14}$.

Os receptores $\mathrm{D}_{2}$ estão acoplados a múltiplos sistemas efetores, sendo sua transdução efetuada por inibição da atividade da adenilciclase, supressão de correntes de $\mathrm{Ca}^{2+}$ e ativação de correntes de $\mathrm{K}^{+15}$. Assim, é plausivel que a inibição crônica de receptores $\mathrm{D}_{2}$ pré-sinápticos causada pela domperidona acarrete aumento do tônus noradrenérgico em determinados territórios, levando, portanto, a prolongado aumento da resistência periférica regional, diminuição do aporte sangüíneo e provável sofrimento das estruturas afetadas.

A dopamina, por sua vez, está contida nas projeções neuronais, substância negra, gânglios basais e hipotálamo de conceptos de ratas já aos 13-15 dias de vida intra-uterina ${ }^{16}$; é a primeira substância neuroativa no curso da sintese de catecolaminas em todo o cérebro ${ }^{17}$.

Poderiamos dizer, então, que, de forma indireta sobre os vasos maternos e direta sobre os vasos fetais, o efeito vasoconstritor determinado pela domperidona poderia explicar o aumento da natimortalidade das crias. Tal fato pode ser deduzido tanto dos nossos resultados quanto dos achados de Marsboon ${ }^{5}$. Embora este autor tenha ministrado o fármaco somente no termo da prenhez, nas doses de 40 e $160 \mathrm{mg} / \mathrm{kg}$, seus dados foram por nós confirmados principalmente no que diz respeito à relação dose-efeito.

Pelo que foi exposto, ainda que não se possa extrapolar os resultados experimentais para a clínica humana, a prescrição de domperidona durante a gravidez deveria ser cercada de critérios extremamente judiciosos.

\section{SUMMARY}

Purpose: the aim of the present work was to study the chronic action of the antiemetic domperidone on the pregnancy of albino rats.

Methods: fifty albino, pregnant Wistar rats were randomly allocated to five groups: GI (control I) = intact rats; GII (control II) = rats receiving the drug vehicle (distilled water) by gavage at the same schedule of the experimental groups; rats in groups GIII, GIV and GV were treated with domperidone by gavage, 2, 6 and $12 \mathrm{mg} / \mathrm{kg}$ per day, respectively, divided into 4 daily doses, always in $1 \mathrm{ml}$ of distilled water, from time zero up to the 20th day of pregnancy. The evolution of body weight gain was followed throughout and the animals were sacrificed at term (20th day) by deep ether anesthesia. Number of fetuses, placenta and implantation sites, placenta and fetus weight, fetal malformations and maternal and fetal mortality were evaluated.

Results: we observed only intrauterine fetal mortality with 14 , 26 and 32 in 74, 60 and 57 newborns of the groups III, IV and , respectively.

Conclusion: though the results of animal experimentation cannot directly be transposed to human conditions, this paper calls attention to the need for a safe judgement when prescribing domperidone to a first-trimester pregnant patient in order to reduce her emetic crises.

KEY WORDS: Drugs in pregnancy. Hyperemesis. Fetal death.

\section{Referências}

1. Fairweather DVI. Nausea and vomiting in pregnancy. Am J Obstet Gynecol 1968; 102:135-75.

2. Soules MR, Hughes CL, Garcia JÁ, Livengood $\mathrm{CH}$ Prystowsky MR, Alexander E. Nausea and vomiting of pregnancy: role of human chorionic gonadotropin and 17-hydro-progesterone. Obst Gynecol 1980; 55: 696-700.

3. Dipalma, JR. Drugs for nausea and vomiting of pregnancy. Am Fam Physician 1983; 28:272-4.

4. Masaki T. Endothelins: homeostatic and compensatory actions in the circulatory and endocrine systems. Endocr Rev 1993; 14:256-68.

5. Marsboom H. Oral embryotoxicity and teratogenic study in Wistar rats (segment III). Beerse: Janssen Pharmaceutical Research Laboratories; 1976. Experiment, 634 (76.01.28).

6. Winick M. Cellular growth in intrauterine malnutrition. Pediatr Clin North Am 1970; 17: 69-75.

7. Edwards CM. Chemotherapy induced emesis mechanisms and treatment: a review. J Roy Soc Med 1988; 81:658-62.

8. Ungerstedt U. Stereotaxic mapping of the monoamine pathways in the rat brain. Acta Physiol Scand Suppl 1971; 367:1-48.

9. Bolton TB. Mechanisms of action of transmitters and other substances of smooth muscle. Physiol Rev 1979; 59:606-718. 
10.Levitt P, Rakic P. The time of genesis, embryonic origin and differentiation of the brainstem monoamine neurons in the rhesus monkey. Brain Res 1982; 256:35-57.

11.Simonson MS, Dunn MJ. The molecular mechanisms of cardiovascular and renal regulation by endothelial peptides. J Lab Clin Med 1992; 119:622-39.

12.Seeman P. Dopamine receptors and dopamine hypothesis of schizophrenia. Synapse 1987; 1:133-52.

13.Laduron PM, Leysen JE. Domperidone a specific in vitro dopamine antagonist, devoid of in vivo central dopaminergic activity. Biochem Pharmacol 1979; 28:2161-5.
14.Tsien RW, Lipscombe D, Madison DV, Bley KR, Fox AP. Multiple types of neuronal calcium channels and their selective modulation. Trends Neurosci 1988; 11:431-8.

15.Vallar L, Meldolesi J. Mechanisms of signal transduction at the dopamine $\mathrm{D}_{2}$ receptor. Trends Pharmacol Sci 1989; 10:74-77.

16.Langer SZ, Lehmann JE. Presynaptic receptors on catecholamine neurons. In: Trendelenburg U, Weiner N, editors. Catecholamines I. Berlin: Springer-Verlag; 1988. p. 419-507. (Handbook of experimental pharmacology, 90).

17.Gilmore D. Neurosecretions and neurotransmitters of the fetal hypothalamus. In: Polin RA, Fox WW. Fetal and Neonatal Physiology. $2^{\text {nd }}$ ed. Philadelphia: WB Saunders; 1998; p.2396. 